\title{
estratégias PARA DETECÇÃo PRECOCE DE PROPENSÃo À EVASÃO
}

\section{(STRATEGIES FOR THE EARLY DETECTION OF EVASION PROPENSITY)}

\author{
Adelina Mezzari \\ Universidade Federal de Ciências da Saúde de Porto Alegre, UFCSPA (Brasil) \\ Liane Margarida Rockenbach Tarouco \\ Barbara Gorziza Avila \\ Geraldo Ribas Machado \\ Rute Vera Maria Favero \\ Universidade Federal do Rio Grande do Sul (Brasil)
}

Ana Marli Bulegon

Centro Universitário Franciscano em Santa Maria, RS (Brasil)

\section{RESUMEN}

A evasão é uma preocupação nos cursos tanto presenciais quanto de EAD e este trabalho apresenta os resultados de uma investigação sobre suas causas. Além da investigação a partir de referências de outros trabalhos sobre o mesmo tema, foi desenvolvido um estudo usando dados derivados das atividades em um curso a distância para formação de professores em serviço. Este estudo e a pesquisa realizada visaram diagnosticar as causas da evasão e buscar indicadores que possibilitem identificar precocemente situações que contribuam para a evasão. A análise dos registros das atividades no curso também avaliou as estratégias de comunicação mediada por computador buscando detectar indícios de risco de evasão, bem como identificar fatores relevantes que levam a evasão. Os dados permitiram elicitar indicadores de atividade dos alunos em cursos EAD os quais permitem identificar situações onde exista risco mais elevado de evasão.

Palabras clave: ensino a distância, evasão, mediação, fórum.

\section{ABSTRACT}

Evasion is a concern for both onsite and D-Ed. courses. This paper presents the results of research about its causes. In addition to investigating references from other works about the same theme, we also developed a study using data derived from distance course learning 
for in-service teachers. This study and the accompanying research aim to diagnose the causes of 'evasion' and to obtain evidence that helps to prevent situations contributing to evasion. There is an analysis of the records of course activities and an evaluation of computerbased communication strategies, i.e. with the aim of detecting evasion risk levels, as well as identifying relevant factors that lead to evasion. These data help to elicit indicators of student activity in distance learning courses, which, in turn, facilitates the identification of situations where there is a higher risk of evasion.

Keywords: distance education; evasion; mediation, fora.

O mundo globalizado trouxe mudanças significativas no trabalho. O trabalhador, neste novo cenário, deve ser um sujeito criativo, crítico e pensante, preparado para agir e se adaptar rapidamente às mudanças dessa nova sociedade, atualizando-se constantemente. As tecnologias da informação e da comunicação (TIC) mudaram a forma de interação entre os indivíduos, tornaram a comunicação mediada por computador (CMC) como uma forma de contato permanente mesmo a distância (Losada, 1999; Martin-Blas y Serrano, 2009; Martin y López, 2012). As TIC também proporcionaram um espaço de ensino e aprendizagem continuada. Sendo assim, a educação sofreu mudanças em seus paradigmas, o conhecimento não é mais compartilhado somente em espaços limitados, mas também mediante o uso das tecnologias da informação e comunicação proporciona maior interação entre professor e aluno. Nos ambientes de aprendizagem informatizados são disponibilizados diversos tipos de comunicação virtual como fóruns, chats, e-mail, cartas, telefone e outros onde todos contribuem para adquirir conhecimentos (Losada, 1999; Heller, Martin, Haneef y Gievska-Karlin, 2001; Jones y Issroff, 2005; Beckett, Amaro-Jiménez y Becketto, 2010; Vercellone, Jablokow y Friedel, 2012).

O crescimento da EAD está acompanhado do aumento no uso da TIC no processo de educação a distância no Brasil conforme destacam as análises de Araujo y Lucena (2005). Segundo a ABED (2013), 179 instituições ofereciam curso a distância em 2011. A quantidade de alunos matriculados no Brasil, passou de cerca de 500.000 em 2009 para mais de 3.500.000 em 2011, segundo relatório da ABED.

Os cursos na modalidade a distância, possibilitam a atualização permanente do trabalhador na atualidade. Alguns destes cursos enfatizam o atendimento ao aluno, criando vínculos e laços afetivos aumentando a probabilidade de continuidade da participação dos mesmos. Apesar de que nesta modalidade de ensino, a dificuldade financeira e falta de tempo são motivos alegados pela maioria dos alunos que abandonam sua formação, observa-se que quanto mais interação e atenção é proporcionada ao aluno, menor é a taxa de evasão, mesmo para os que estão geograficamente dispersos. O diálogo pode ser uma variável que contribui 
para este resultado e para uma aprendizagem mais significativa conforme destacam diversos autores (Dingus y Ellis, 2005; Fidas, Komis, Tzawavaris y Avouris, 2005; De Wever, Schellens, Valck y Van Keer, 2006; Regan 2012; Costa, 2013; Dowden, 2013). No presente trabalho foram investigados indicadores que possam elicitar esta interrelação entre quantidade e e qualidade da atenção dispensada ao aluno e a probabilidade de sua evasão.

A evasão tem sido a grande preocupação entre os responsáveis por instituições de ensino em geral, sendo um problema internacional, inclusive no Brasil (GrauValldosera y Minguillón, 2011; Baxter, 2012; Fiuza, 2012; Cheng, Kulkarni y Klemmer, 2013). A ocorrência da situação em que estudantes iniciam mas não terminam seus cursos gera desperdícios sociais, acadêmicos e econômicos (Tinto, 1975; Lobo, Montejinas, Hipólipo y Lobo, 2007; Park y Choi, 2009; Nistor, 2010; Leeds, 2013).

Para tentar minimizar esta realidade, torna-se necessário investigar e elicitar os fatores mais estreitamente relacionados com o desempenho acadêmico do estudante de EAD e em particular com a evasão. Estratégias para modificar a situação indesejada de altas taxas de evasão certamente passam por avaliar a estratégia pedagógica. Que o aluno deve participar ativamente na construção de seu aprendizado e o professor não pode ser expositor ou facilitador, é um resultado amplamente destacado e com significativa fundamentação teórica (Tinto, 1987; Losada, 1999; Moore y Kearsley, 2007). Tanto no modo presencial ou a distância, o professor deve criar situações e condições de aprendizagem adequadas, como fator decisivo para alcançar as mudanças desejadas no paradigma psico-pedagógico (Fredrickson, 1998; Losada, 1999). Estas mudanças implicam na substituição de paradigmas educacionais mais calcados em exposição de conhecimento por paradigmas que sejam apoiados em outros mecanismos de instigação do processo de aprendizagem que envolvam uma participação mais intensa e ativa do estudante (Flach y Antonello, 2011). O ciclo de Kolb oferece uma estratégia para organizar a atividade de ensino-aprendizagem com vistas a ampliar a participação ativa do estudante (Kolb y Fry, 1975). 


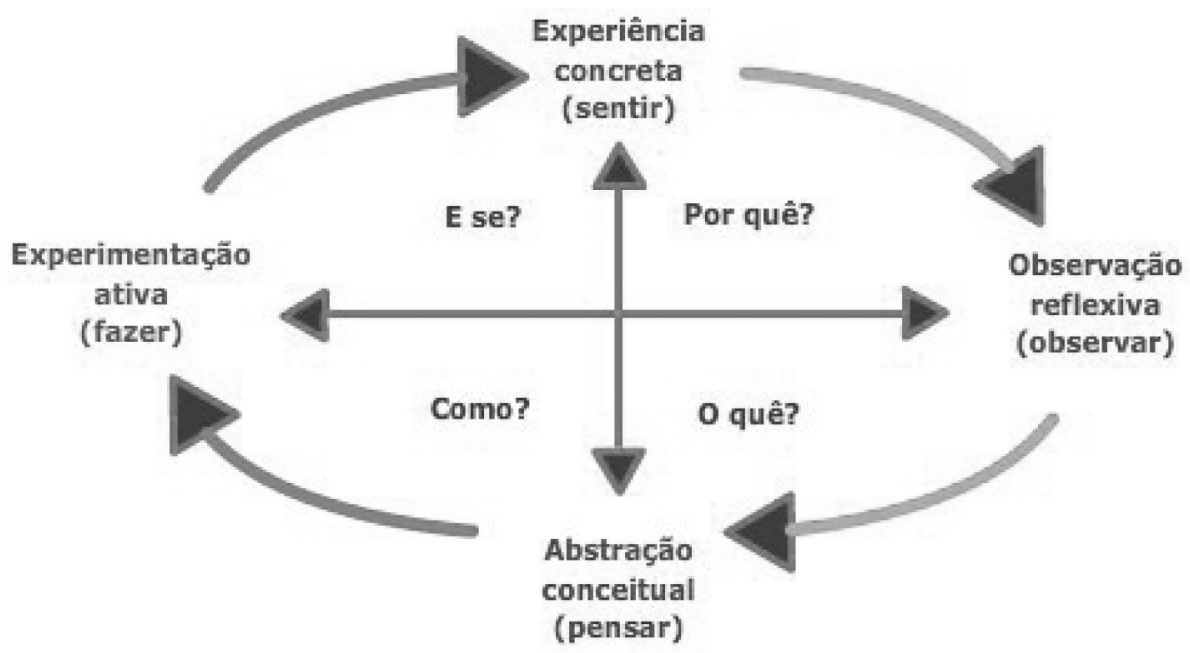

Figura 1. Ciclo de Kolb

A partir desse modelo, McCarthy (1986) propõe um sistema de aprendizagem, contemplando os quatro tipos de aprendizados propostos por Kolb (1984), no qual foram incorporadas perguntas que instigam o ensino-aprendizagem. McCarthy (1986), afirma que a aprendizagem ocorre pela passagem dos quatro quadrantes deste ciclo. Sendo assim, a experiência induz à necessidade da aprendizagem, a qual requer uma observação reflexiva da experiência e a consolidação de conceitos para integrar a nova experiência com o já conhecido. Após a integração, é realizada a avaliação e essa ação resulta em novas experiências concretizando assim a aprendizagem por meio de um ciclo.

O Modelo proposto por Kolb diz que idealmente este processo representa um ciclo ou espiral de aprendizagem pelo qual o estudante deve passar, envolvendo atividades de experimentação, reflexão, pensamento e ação. As experiências concretas levam a observações e reflexões. Dependendo do estilo de aprendizagem de cada pessoa ela pode preferir iniciar o processo de aprendizagem através da experimentação ou através de novas informações e refletir sobre as mesmas.

Em um contexto de educação a distância, o processo de ensino aprendizagem precisa diferir da abordagem tradicional de sala de aula que usualmente inicia pela fase de apresentação de conteúdos sendo baseada na conceitualização abstrata e depois, com a condução do professor vai sendo direcionada para a experimentação ativa pelos estudantes com exercícios, estudos de casos e outros. Uma experiência concreta nem sempre é possível por questões de tempo (duração da aula) e local 
(não disponibilidade de laboratório ou dos elementos necessários para a experiência concreta). $\mathrm{O}$ retorno para a fase de experiência concreta em um momento posterior de reunião do grupo pode ser a oportunidade para que as reflexões derivadas da observação ocorram. Em um curso a distância o início pela etapa de conceitualização pode desestimular o aluno face à natural dificuldade que o maior nível de abstração impõe. Todavia o aluno de cursos a distância tem uma bagagem de vida maior e suas experiências pessoas podem ensejar análise e reflexão seguidas de teorias que permitam explicar, justificar modos de atuar no mundo real e, a seguir, novas experimentações podem ser desencadeadas e o ciclo reiterado com reflexão, abstração e novas alternativas para experimentação ativa (Underwood, 1998).

Sabe-se que somente o professor não consegue obter sucesso no processo de ensino e aprendizagem se o aluno não for motivado para tal. Sendo o aluno o centro do processo de aprendizagem, todos os métodos de ensino-aprendizagem devem estar voltados para ele. O aprender é um processo ativo e neste sentido deve ser pautado por uma contínua busca de soluções para resolver problemas. Assim como o desenvolvimento intelectual das crianças ocorre em função das interações sociais (Vygotsky, 2008) no caso de educação de adultos as interações sociais são também elementos básicos na reflexão e capazes de fornecer ao aprendiz maneiras de tornar mais eficazes seus esforços de adaptação e solução de problemas. $\mathrm{O}$ aluno contribui com seu aprendizado ao enviar por meio de mensagens seus pensamentos e idéias, dialogando com seu professor e com seus colegas também. Cria-se assim uma rede de aprendizagem que amplia a zona de desenvolvimento proximal conforme defendido por Vygotsky (2008) que amplia a capacidade de resolução de problemas derivada da colaboração com colegas mais capacitados. O saber resulta da mediação dialógica pela troca de informações, conteúdos e afeto e a palavra é a origem desta comunicação (Tinto, 1993; Freire, 2006).

A participação ativa do aluno na construção do conhecimento é imprescindível e para que isso ocorra, é necessário estabelecer condições que dêem suporte à esta estratégia construtivista de aprendizagem. O professor deverá auxiliar os alunos a organizarem seus caminhos na aprendizagem, disponibilizando múltiplas opções sobre os fenômenos e problemas estudados, permitindo-lhes que tenham soluções alternativas e testem suas decisões. Permitir que a aprendizagem tenha contextos realistas e relevantes também é importante e neste sentido a aprendizagem pode e deve ser organizada com base em experiências sociais do professor com os alunos e entre os alunos, encorajando assim a meta-aprendizagem (Tarouco, Hack, Vit y Geller, 2000). 
No Brasil o Instituto Nacional de Estudos e Pesquisas Educacionais (INEP), órgão do Ministério da Educação do Brasil, tem divulgado dados de ingressantes e egressos do ensino superior o que possibilita o estudo da evasão nos cursos superiores do Brasil. Com estes dados o Anuário Estatístico Brasileiro de Educação a Distância (ABRAEAD, 2007) verificou que aproximadamente a metade dos alunos, $48 \%$, que ingressam anualmente no sistema de ensino não obtêm a titulação no prazo previsto para a integralização dos estudos. Em outros países a situação de abandono apresenta números tais como: México - 31\%, Japão - 7\%, Alemanha - 30\%, Estados Unidos - 34\%, França - 41\%, Itália - 58\%, Suécia - 52\% e Reino Unido 17\%. Na América do Sul tem-se dados como os do Chile - 54\%, Venezuela - 52\% e o Uruguai - 72\% (Faria, Alcantara y Vasco, 2008). Entretanto, a situação vem sendo amenizada, conforme apontam os dados do ABRAEAD (2008), pois no universo de $60 \%$ das instituições pesquisadas, $11 \%$ vêm apresentando índices maiores do que $30 \%$ de alunos evadidos. Trata-se de uma queda considerável nos índices de evasão, porém, ainda vem sendo desperdiçado muito dinheiro público em decorrência de tais desistências. Os resultados publicados pela ABED (2013), referentes à evasão em cursos no Brasil durante o ano de 2011, mostram a evasão percentual média indicada na tabela 1.

\begin{tabular}{|l|c|c|}
\hline Tipo de curso & $\begin{array}{c}\text { Quantidade } \\
\text { de cursos }\end{array}$ & $\begin{array}{c}\text { Evasão média } \\
(\%)\end{array}$ \\
\hline Autorizado & 81 & 20,5 \\
\hline Disciplinas & 25 & 17,6 \\
\hline Livres & 73 & 23,6 \\
\hline Corporativos & 33 & 20 \\
\hline
\end{tabular}

Tabela 1. Evasão por tipo de curso em 2011

Cordero (2005) relata sobre o sistema aplicado na Espanha que o aluno é muito exigido quanto aos conteúdos teóricos, sendo a boa formação uma certeza. Apesar disso ocorre um alto índice de desistência, que chega a 80\% entre os alunos matriculados nos dois primeiros anos. Uma das razões de tão alta evasão ocorre porque na Espanha são poucos os cursos a distância oferecidos e quase todos pela UNED. Se o aluno gostou do curso ele continua, caso contrário não dispõe de muitas outras opções. Esse monopólio pode ser o responsável por este alto percentual de evasão referido. Lassibile y Gómez (2007) também tentaram verificar os motivos do abandono dos estudantes no ensino superior na Espanha. A preparação acadêmica foi um dos principais fatores que influenciou sobre os alunos. Além disso os alunos mais velhos, a entrada mais tarde no ensino superior e o apoio financeiro também foram fatores relevantes. Park y Choi (2009) ressaltaram em sua pesquisa a necessidade de 
assegurar apoio organizacional, elicitar a relevância do curso e a satisfação do aluno com o mesmo. Os resultados de Parks sugeriram que é possível reduzir a evasão se os desenvolvedores do curso e seus instrutores encontrarem maneiras de melhorar a relevância do curso. Até sistemas de previsão de evasão baseados em aprendizagem de máquina foram desenvolvidos com vistas a tentar antecipar tal como relatado por Lykourentzou (2009).

Face a este cenário, percebe-se que há necessidade constante de investigações que permitam diagnosticar as causas da evasão e buscar indicadores que possibilitem identificar precocemente situações que contribuam para a evasão. $O$ presente estudo visa avaliar na comunicação mediada por computador das comunidades virtuais, estratégias para detectar indícios de risco de evasão, bem como identificar fatores relevantes que levam à evasão dos alunos na Educação a Distância (EAD). Neste sentido, inicialmente, serão apresentadas as características relevantes de comunidades virtuais que constituem o contexto básico onde ocorre a EAD.

\section{COMUNIDADES VIRTUAIS}

As comunidades virtuais são agregações sociais e culturais que emergem na Internet, formadas pelo encontro sistemático de um grupo de pessoas. O fluxo de informações gerado nas Comunidades virtuais oportuniza aos participantes ampla troca de conhecimento através de atividades baseadas num compartilhamento interativo, além da riqueza proporcionada pelas discussões estabelecidas nestes ambientes (Hung y Cheng, 2013). No âmbito das comunidades virtuais em geral, ocorre aprendizagem num contexto de educação continuada, às vezes de forma menos organizada. No âmbito de $\mathrm{EAD}$, pode-se considerar que cada grupo de aprendizagem constituído (turmas, sub-grupos e outros) constitui também uma comunidade virtual. Os Ambientes Virtuais de Aprendizagem (AVAs) são espaços que oferecem recursos para apoiar o Diálogo e a Comunicação mediada pelo computador (CMC) ou Computer Supported Colaborative Learning (CSCL/CSCW), essencial para o funcionamento de uma comunidade virtual, além de outros recursos voltados a apoiar o processo de ensino e a aprendizagem como um todo (Tarouco, 2004; Girasoli y Hannafin, 2008).

O diálogo nas Comunidades virtuais é sustentado pelos fóruns de discussão, chat e outras ferramentas, que contribuem para potencializar a interatividade entre os alunos em atividades problematizadoras, assíncronas e síncronas, que ensejam a participação e a comunicação entre todo o grupo em questão incluindo até mesmo um ambiente baseado em realidade virtual que amplie a sensação de presencialidade nas interações remotas (Tarouco, Vicari, Geller y Meirelles, 2003; Lima y Tarouco, 
2005). O diálogo tem tanto valor no suporte à atividade da comunidade e aprendizagem que até mesmo o material educacional de apoio pode ser organizado em uma base de conhecimento com o acesso ao mesmo sendo estabelecido a partir de questões apresentadas pelo alunos, em linguagem natural, as quais podem ser processadas por um chat-robot gerando respostas que tentam assemelhar-se às produzidas por humanos interagindo tal como descrito por Leonhardt, Tarouco, Vicari, Santos y Silva (2007); Tarouco (2004). O fórum, por oferecer possibilidades de construção coletiva do conhecimento de forma assíncrona, tornou-se a ferramenta mais frequentemente usada nas atividades de ensino-aprendizagem, permitindo aos alunos a comunicação vertical (com professor e tutores) e horizontal (com seus pares) durante todo este processo.

Nas comunidades virtuais de ensino aprendizagem os Ambientes Virtuais de Aprendizagem (AVAs) são os espaços que apoiam o processo da aprendizagem e tem como premissa a virtualização (Jones e Issroff, 2005; Masiello, Ramberg y Lonka, 2005). Os AVAs tipicamente incluem funcionalidades para apoiar a comunicação mediada por computador e a disponibilização de material nos cursos on-line. É um novo espaço de relacionamento que tem características próprias e é preciso aprender a usá-lo para auferir benefícios (Vit y Tarouco, 200o; Jones e Issroff, 2005). A proficiência no uso das ferramentas do AVA, tem impacto na motivação dos integrantes das comunidades para permanecer e atuar produtivamente. Isto é verdade em qualquer ambiente de suporte para comunidades virtuais. Se o uso das funcionalidades de tais ambientes for complexo ou mesmo em sendo simples não for dominado por seus usuários no mínimo teremos subutilização das funcionalidades e conseqüente perda de eficácia pelo não aproveitamento das mesmas. A evasão também pode ser motivada pela sensação de impotência para usar o ambiente e esta dificuldade foi constatada em diversos cursos a distância trabalhados pelos autores. Em um caso particular analisado anteriormente, uma das causas de evasão relatadas foi a dos problemas tecnológicos que incluíam a dificuldade para acessar e usar o ambiente virtual de aprendizagem (Lima y Tarouco, 2004). Embora atualmente o nível de alfabetização das pessoas tenha avançado bastante, ainda se percebe este tipo de dificuldade por parte dos participantes de curso a distância, especialmente quando os estudantes são originários de locais mais distantes dos grandes centros. Embora tenham condições e conhecimento para navegar pela Internet, enviar e receber mensagens ou mesmo participar de redes sociais muitos encontram dificuldade quando necessitam anexar um ou mais arquivos a uma mensagem ou enviar arquivos. Uma pesquisa realizada pelo Cetic.br (2012) mostrou que os professores indicaram como possíveis obstáculos para o uso da tecnologia na educação, a ausência de suporte técnico, falta de conhecimento sobre o computador 
e Internet, velocidade de conexão insuficiente e falta de confiança nas informações contidas na Internet.

\section{Diálogo e a Comunicação Mediada por Computador (CSCL/CSCW)}

O professor é um contraponto imprescindível para que a relação dialógica se torne pedagógica e produza aprendizagem. Para Freire (2003), pode haver diálogo em uma aula expositiva ou em uma palestra que leve os educandos à reflexão. Ele afirma que "dialogar não é tagarelar. Por isso, pode haver diálogo na exposição crítica, rigorosamente metódica, de um professor a quem os alunos assistem não como quem come o discurso, mas como quem aprende sua intelecção”.

No ensino, é necessário reciprocidade, para que os alunos encontrem no professor um pouco de si e resulte no aprender (Martin-Blas y Serrano, 2009; Reagan, 2012; Dowden, 2013). Podem ocorrer na forma síncrona ou assíncrona em ambientes virtuais de aprendizagem, através de textos ou mensagens por e-mail, chats, fóruns, cartas, telefonemas e outros (Girasoli y Hannafin, 2008; Offir, Lev y Bezale, 2008; Costa, 2013). A comunicação através do diálogo se realiza através da linguagem, resultando em comunicação e intercâmbio social (Underwood y Underwood, 1998; Walraven, Brand-Gruwel y Boshuizen, 2009). O diálogo quando mediado por computador usa uma linguagem específica através de textos escritos. A ocorrência de diálogo entre o professor e alunos e entre alunos num AVA, colabora para a permanência dos alunos dentro desse ambiente. Havendo o diálogo entre eles, a evasão pode diminuir nesses ambientes, pois o aluno não se sente só neste processo de aprendizagem, e mesmo geograficamente distantes, esta interação aproxima os participantes (Fávero y Franco, 2006). O professor ao dialogar com seu aluno vivencia momentos únicos de afeto e construção mútua do conhecimento tanto por parte dele como do aluno e contemplando, desta forma, tanto o aspecto cognitivo quanto o afetivo de ambos (Sapiro, Feltovich, Jacobson y Coulson, 1995; Mazzolini y Madison, 2007).

A Comunicação Mediada por Computador é uma estratégia que apoia a participação ativa do aluno na construção do seu conhecimento. O CSCL/CSCW oferece um novo modelo de ensino e aprendizagem possibilitando a interação e a auto-aprendizagem. A participação dos alunos, com seus diferentes conhecimentos, níveis de experiência, competências e habilidades em comunidades criadas neste modelo de ensino, permite a construção do conhecimento de forma coletiva (Heller, 2001; Jones e Issroff, 2005; Costa, 2013). O estudo realizado por Fidas, Komis, Tzawavaris y Avouris (2005) revelou que ao contrário da expectativa, um grupo de alunos mais heterogêneos produziram soluções com qualidade similares ao de 
um grupo referência, sendo assim concluíram que o ambiente deve ser motivador e instigar o aluno a procurar mais conhecimentos.

\section{EVASÃO}

Evasão é o ato da desistência em qualquer momento do curso (Fredrickson y Losada, 2005). A evasão é uma preocupação nos cursos tanto presenciais quanto de EAD. A busca de suas causas é objeto de trabalho e muita pesquisa educacional. $\mathrm{O}$ aluno de EAD precisa organizar-se para as atividades pois não há uma rotina acadêmica imposta como no caso dos cursos presenciais. A liberdade para realizar suas atividades nos momentos mais convenientes enseja a procrastinação que leva ao acúmulo de atividade até um ponto onde nada mais resta ao aluno a não ser desistir. A falta do convívio com os colegas que poderia servir de motivação para a continuidade no curso, leva a um sentimento de isolamento, solidão que desmotiva o aluno de EAD. Sendo assim um fator de desistência é a cultura do aluno quando não assume o curso efetivamente se não tiver a cobrança de sua empresa ou um compromisso pessoal consigo mesmo (Donald, 2002; Morin, 2003). Isto pode ser constatado a partir de comentários iniciais recebidos de alunos participantes de cursos para os quais foram enviados por determinação de suas coordenações e cujo desempenho desde o início foi minimalista, culminando com alta taxa de evasão. Uma pesquisa sobre motivos de evasão, no presente estudo, evidenciou como causa preponderante a falta de tempo, tal como evidencia a tabela 2.

\begin{tabular}{|l|l|}
\hline \multicolumn{1}{|c|}{ Categorias - evadidos } & Quantidade \\
\hline Falta de adaptação à EAD (1) & 1 \\
\hline Apresentação do conteúdo 2) & 1 \\
\hline Dificuldade de interação com tutor (3) & 2 \\
\hline Excesso de conteúdo (4) & 2 \\
\hline Falta de interesse (5) & 3 \\
\hline Falta de tempo (6) & 14 \\
\hline Organização pessoal (7) & 5 \\
\hline Outro curso (8) & 3 \\
\hline Problemas administrativos (9) & 1 \\
\hline Problemas de saúde (10) & 8 \\
\hline Problemas tecnológicos (11) & 7 \\
\hline Sem resposta & 60 \\
\hline
\end{tabular}

Tabela 2. Motivos de evasão e ocorrência 
O motivo "organização pessoal" embora distinguido da falta de tempo, reflete também a questão da falta de tempo. Cabe observar que o aluno que busca na educação à distância uma formação básica ou continuada, usualmente tem outros encargos profissionais ou domésticos que dificultam sua participação com dedicação maior, mas destaque-se isto ocorreria também em um curso presencial e sem a opção do curso a distância, esta pessoa não teria qualquer opção de formação ou educação continuada.

Problemas de saúde (doenças pessoas ou na família) também constituíram motivo freqüente apresentado como justificativa para a evasão.

Um fator externo conhecido sobre a população típica de educação a distância é a idade do alunos, constatando-se maior probabilidade no acúmulo de atividades profissionais, domésticas e acadêmicas. Nesta população, constituindo uma sobrecarga demasiada e diminuindo a capacidade para alcançar todos os objetivos almejados ocasionando desistência. Este mesmo fator externo, pode explicar uma outra causa de evasão declarada que foi derivada de problemas de inabilidade para usar a tecnologia como recurso e apoio no curso desenvolvido à distância. Usualmente constata-se que alunos mais jovens tem inata habilidade para usar a tecnologia e se desincumbem naturalmente das tarefas realizadas no ambiente virtual de aprendizagem. Em oposição, para o grupo da faixa etária mais elevada, são mais freqüentes as dificuldades no uso do ambiente com problemas que variam desde esquecimento de senha e a falta de compreensão das orientações relativas ao uso dos recursos de ambiente e outros.

A comunicação intermediada por computador ocorre de forma escrita e tradicionalmente a escrita tem sido encarada como um forma de expressão mais tradicional e oficial. Em decorrência muito cuidado precisa ser tomado na redação das mensagens para os alunos de EAD pois a ênfase pretendida para uma mensagem pode não ser bem compreendida e ser distorcida levando a dificuldades de comunicação, sedo um outro, dos fatores de evasão reportados. Amenizar as colocações escritas, mediante o uso de recursos gráficos (smileys) e outras formas de redação constituem estratégias para diminuir a imponência de uma comunicação que deva ser mais coloquial e amistosa. A comunicação pessoal do professor ou tutor com os alunos tem importante efeito na redução da evasão pois é capaz de aumentar a motivação e levar os participantes a se esforçarem para acomodar todas as demandas de tempo que competem com a dedicação ao curso. Até mesmo o tempo decorrido entre uma manifestação do aluno e a resposta do tutor ou professor pode ser assumido pelo aluno como descaso e contribuir para reduzir a motivação e aumentar a tendência para a evasão. 
Outros fatores que também podem resultar em evasão são o desinteresse pela continuidade dos estudos, priorização de outras atividades profissionais ou mesmo acadêmicas e a falta de adequação ao modelo de aprendizagem da EAD que implica em maior autonomia, maior necessidade de gestão de múltiplos fatores que concorrem pela atenção do aluno e impõem obstáculos à sua dedicação ao curso. Diversos autores citam motivos para evasão tais como falta de encontros presenciais, a reprovação, baixa estima do estudante, estratégias instrucionais inadequadas, uso inadequado da tecnologia e desrespeito com o aluno (Fávero y Franco, 2006; Santos, Tomake, Oliveira Neto, Cazarini, Araújo y Oliveira, 2008; Kingston, 2008; Park y Choi, 2009; Nistor y Neubauer, 2010).

Neste artigo, após análise teórica e estudo de caso visando elicitar as causas majoritárias de evasão em EAD, foi realizada uma investigação com vistas a investigar indicadores que pudessem sinalizar tendências para evasão. Foram analisados dados referentes à participação de aproximadamente 230 alunos que freqüentaram um curso de EAD com duração de 8 meses e os resultados são comentados na próxima sessão.

\section{INDICADORES DA PARTICIPAÇÃO DOS ALUNOS NUM AVA}

A participação dos alunos em ferramentas dos AVAs pode ser um indicador para detectar alunos prestes a evadir. Inicialmente, o estudo buscou validar alguns indicadores de desempenho, propostos por Araújo e Lucena (2005). Neste trabalho são usados alguns índices, originalmente propostos por Losada (1999), para uso na avaliação de desempenho de equipes. Estes índices permitem avaliar as interações entre os participantes e correlacionar os valores obtidos com o desempenho da equipe. O trabalho de Araújo e Lucena (2005) foi focado na avaliação de comunidades virtuais de aprendizagem. Os índices descritos por êles para avaliar os fóruns, denotam o tipo de interações entre os alunos e professor. Foram definidos dois tipos de Interação (TI) que representam o sentido ou direção das interações no fórum, horizontal ou vertical. A participação horizontal é aquela dirigida de aluno para aluno, de aluno para todos e do professor para todos os alunos. Na participação vertical ocorre a comunicação do aluno para o professor e do professor para um único aluno. A TI é obtida dividindo o total de comunicações horizontais $(\mathrm{H})$ pelo total de comunicações verticais (V). Um valor menor para o indicador TI denota um relacionamento mais verticalizado entre os participantes com a comunicação ocorrendo em maior freqüência entre o tutor/professor e os alunos. A falta de uma maior parcela de comunicação horizontal pode indicar uma passividade maior dos alunos, quando limitam-se a responder questionamento que lhe são diretamente endereçados ou maior dependência em relação ao professor, quando participam 
do fórum primordialmente para dirimir junto ao professor dúvidas conceituais ou operacionais (relativas a trabalhos do curso). A participação mais significativa, capaz de denotar atividade coletiva de construção de conhecimento seria representada por um valor do indicador TI mais elevado.

Na qualidade das postagens no fórum foi representada pelo indicador denominado Tipos de Participação (TP) combinado com o de Comunicação (TPC). As contribuições dos alunos são categorizadas como:

- A - o aluno não contribui para a discussão em pauta.

- B - somente responde a alguma pergunta do professor.

- C - resposta questionadora com dilemas, alternativas e solicita posicionamentos.

- D - debate, comenta contribuições anteriores com propriedade, responde questionamento de colegas, contra-argumenta.

- E-a contribuição é sintetizadora onde ajusta, adapta e elabora parecer conclusivo.

O indicador TPC é obtido dividindo a soma do totais de participações to tipo C,D,E pela soma das participações to tipo A,B. Neste índice, espera-se que quanto maior o TPC, maior será a construção do conhecimento pois são as contribuições do tipo C, D e E que evidenciam indícios de pensamento crítico.

Por fim, o indicador $\mathrm{P} / \mathrm{N}$ é definido pela relação entre manifestações que evidenciam Positividade (P) e as que evidenciam negatividade (N). Como positivas são consideradas frases ou orações com características como saudação inicial ou final, sugestões para correções de problemas, respostas, contribuições, perguntas com ações positivas, otimismo, elogios e sinais de aprendizagem com a ferramenta. Como negativas são consideradas frases ou orações relacionadas a ações negativas, problemas sem vontade ou possibilidade de resolvê-los, pessimismo, juízos negativos, cinismo, desconhecimento da participação anterior do colega. Losada (1999) demonstrou que a relação $\mathrm{P} / \mathrm{N}$ é tão importante quanto a conectividade para determinar o desempenho de uma equipe. Fredrickson y Losada (2004) determinaram matematicamente o valor de $\mathrm{P} / \mathrm{N}$ em 2,9013. Losada (1999) estudou as relações entre manifestações positivas e negativas em equipes de alta e baixa performance. Este limiar determinado por ele permite identificar nas interações de um grupo aqueles que são capazes de uma compreensão complexa dos demais. Um grupo no qual a relação entre as manifestações positivas e as negativas esteja acima 
do valor 2,9013 desabrocha e evolui enquanto que em outro grupo, onde a relação $\mathrm{P} / \mathrm{N}$ estiver abaixo deste limiar definha mergulhado em justificativas para a falta de realizações.

Este valor determina o desempenho de uma equipe, e o chamaram de "Losada Line". Portanto espera-se que toda equipe que apresentar um valor igual ao superior a 2,9013 deve estar envolvida em uma dinâmica que conduza ao sucesso e ou alto desempenho na construção do conhecimento. Um índice baixo, onde as manifestações negativas são mais freqüentes denota uma dinâmica encontrada em equipes nas quais não ocorreu um bom desempenho.

No presente trabalho buscou-se estabelecer relação entre estes tipos de indicadores e a taxa de evasão, tal como será detalhado na sessão seguinte.

\section{METODOLOGIA DE PESQUISA UTILIZADA}

A metodologia usada no presente estudo avaliou o comportamento observável derivado da participação dos alunos, no uso da ferramenta fórum. Foram selecionados para análise dois fóruns. Estes fóruns refletem interações típicas de participantes de um curso para professores de ensino fundamental desenvolvidos em modalidade de educação a distância. O curso teve duração de 8 meses sendo o fórum a ferramenta principal de interação entre tutores e estudantes. A participação dos alunos no fórum foi considerada também para fins de avaliação. $O$ foco deste curso foi oferecer formação continuada para capacitar professores da educação básica no uso das Mídias em atividades pedagógicas. Os dois fóruns analisados tiveram como objetivo elicitar conceitos e idéias derivadas de reflexão individual e colaborativa sobre dois tópicos relevantes abordados no curso: "Uso de TV e Vídeo no processo educacional" e "Refletindo sobre mudanças". O curso iniciou com 235 alunos. Deste total 107 não concluíram, resultando uma taxa de evasão de $46 \%$. Os motivos apresentados pelos alunos que não concluíram o curso foram os da tabela 1.

Para tentar identificar fatores relevantes da atividade dos alunos foram avaliados indicadores de participação quantitativa e qualitativa baseados no índices propostos por Losada (1999). e também, usados por Araújo e Lucena (2005). Com os alunos que desistiram do curso foi feito contato por telefone e e-mail para identificar os motivos que os levaram à evasão. Embora não tenha sido possível o contato com todos os alunos evadidos, pois dos 107, foi conseguida realimentação sobre os motivos de 47 participantes, pode-se considerar que a amostragem foi relevante. Esta observação ocorreu pela análise desses índices e a categorização das respostas dos contatos com os alunos evadidos os quais permitiram medir a correlação entre os mesmos e a taxa 
de evasão com vistas a elicitar aspectos observáveis no comportamento de turmas participantes de cursos a distância. Estes dados permitem diagnosticar situações em que exista maior probabilidade de evasão e propor alternativas remediadoras.

\section{ANÁLISE DOS RESULTADOS}

Os resultados obtidos da avaliação da participação dos alunos das 5 turmas avaliadas nos dois fóruns analisados calculando os índices TI, TPC e P/N anteriormente descritos e baseados nos trabalhos de Losada (1999) e de Araújo e Lucena (2005) estão apresentados na tabela 3.

\begin{tabular}{|l|l|l|l|l|l|l|}
\hline \multicolumn{1}{|c|}{ Índices } & \multicolumn{1}{|c|}{ T1 } & \multicolumn{1}{|c|}{ T2 } & \multicolumn{1}{|c|}{ T3 } & T4 & \multicolumn{1}{|c|}{ T5 } & TOTAL \\
\hline Total de alunos na turma & 52 & 39 & 50 & 46 & 48 & 235 \\
\hline Total de postagens dos alunos & 209 & 131 & 154 & 98 & 77 & 592 \\
\hline Contribuições significativas & 122 & 64 & 65 & 55 & 41 & 306 \\
\hline Postagens por aluno & 4,02 & 3,36 & 3,08 & 2,13 & 1,60 & \\
\hline Postagens significativas por aluno & 2,35 & 1,64 & 1,30 & 1,20 & 0,85 & \\
\hline Relação P/N & 5,71 & 31,25 & 8,25 & 96 & 78 & 592 \\
\hline Taxa de evasão (\%) & 46 & 53 & 44 & 28 & 64 & 47 \\
\hline Média da turma (o-10) & 4,87 & 5,45 & 5,20 & 6,81 & 1,98 & \\
\hline
\end{tabular}

Tabela 3. Dados derivados da atividade das turmas

Uma análise dos resultados mostrou as seguintes correlações entre os índices de participação dos alunos e resultados como a média alcançada e a taxa de evasão, expressos no gráfico 1 e tabela 4 . 




Gráfico 1. Correlação entre total de postagens e o desempenho

\begin{tabular}{|l|c|c|c|c|c|}
\hline \multicolumn{1}{|c|}{ Tabela com os valores de correlação } & T1 & T2 & T3 & T4 & T5 \\
\hline $\begin{array}{l}\text { Correlação entre total de postagens pelos alunos e a } \\
\text { nota }\end{array}$ & 0,70 & 0,40 & 0,67 & 0,43 & 0,71 \\
\hline $\begin{array}{l}\text { Correlação entre postagens significativas dos alunos e } \\
\text { a nota }\end{array}$ & 0,58 & 0,58 & 0,49 & 0,41 & 0,68 \\
\hline
\end{tabular}

Tabela 4. Valores de correlação entre total de postagens e o desempenho

Pelos resultados é possível constatar que existe uma correlação positiva e significativa entre a intensidade da participação global dos alunos e o resultado que alcançam em termos de desempenho acadêmico. Considerando que o fracasso no curso foi um dos motivos identificados para evasão, percebe-se que ao participar ativamente do curso o aluno não apenas cresce em termos de aprendizagem, pela oportunidade de reflexão colaborativa com o grupo, mas também lança a base para seu crescimento cognitivo que o levará a alcançar melhores resultados e, em conseqüência, o risco de evasão é minimizado. A intensidade da participação do aluno no fórum tem forte correlação com a evasão, conforme pode ser observado no gráfico 2 e tabela 5 . 


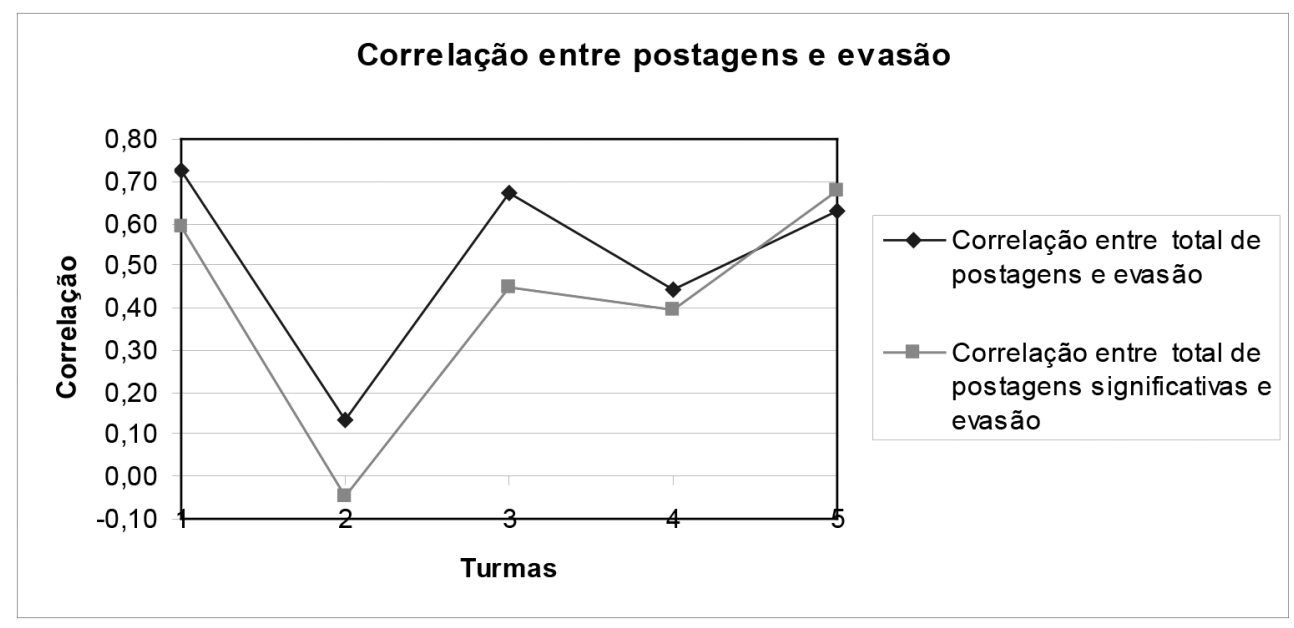

Gráfico 2. Correlação entre total de postagens e evasão

\begin{tabular}{|l|c|c|c|c|c|}
\hline Tabela com a correlação de postagens & T1 & T2 & T3 & T4 & T5 \\
\hline $\begin{array}{l}\text { Correlação entre total de postagens pelos } \\
\text { alunos e a taxa de evasão }\end{array}$ & 0,72 & 0,13 & 0,67 & 0,44 & 0,63 \\
\hline $\begin{array}{l}\text { Correlação entre postagens significativas dos } \\
\text { alunos e a taxa de evasão }\end{array}$ & 0,59 & $-0,05$ & 0,45 & 0,40 & 0,68 \\
\hline
\end{tabular}

Tabela 5. Valores de correlação entre total de postagens e evasão

Por estes dados é possível concluir que a taxa de postagens por aluno pode ser usada como indicador para predizer a probabilidade de evasão pois na maioria das turmas constatou-se elevada correlação tanto entre o total de postagens e a evasão quanto entre o total de postagens significativas e a evasão. O gráfico 2 e a tabela 5 mostram que, exceto no caso na Turma 2 esta forte correlação foi evidenciada. Portanto, a quantidade de contribuições, seja do tipo significativa ou não, pode servir como um indicador precoce de probabilidade de evasão. Ou seja os alunos que não contribuem no fórum são os mais propensos à evasão e um trabalho remediador, de atenção especial por parte do tutor ou mesmo pela coordenação do curso deve ser realizado com vistas a realinhar o aluno para uma direção diferente daquela que leva ao abandono do curso. $\mathrm{O}$ aluno que percebe a atenção da equipe na sua atuação sente-se mais motivado a priorizar o curso frente às múltiplas ocupações que demandam seu tempo. As questões sócio afetivas tem profundo impacto no desempenho acadêmico conforme descrito por Fredrickson (1998) cujo trabalho serviu de base para os índices propostos por Losada (1999). 
Cabe salientar, que na investigação de outros fatores que pudessem influenciar o desempenho das turmas, com taxa de evasão mais alta e mais baixa foi analisado o eventual impacto das condições econômicas da região. Porém o que se constatou foi que, a renda per capita no município de onde eram provenientes a maioria dos estudantes da turma 5 (com 64\% de evasão) era 4 vezes a renda per capita do município que sediava a turma 4 que teve a menor evasão ( $28 \%$ ). O impacto da relação $\mathrm{P} / \mathrm{N}$ e a taxa de evasão foi avaliado a partir dos dados consolidados na tabela 6:

\begin{tabular}{|l|c|c|c|c|c|}
\hline \multicolumn{1}{|c|}{ Relação P/N e evasão } & T1 & T2 & T3 & T4 & T5 \\
\hline P/N & 6 & 31 & 8 & 96 & 76 \\
\hline \% evasão & 46 & 54 & 44 & 28 & 65 \\
\hline $\begin{array}{l}\text { Correlação entre a relação P/N das turmas e a } \\
\text { taxa de evasão }\end{array}$ & $-0,166$ & & & \\
\hline
\end{tabular}

Tabela 6. Relação P/N e a taxa de evasão

A relação entre as manifestações positivas e negativas $(\mathrm{P} / \mathrm{N})$ e a taxa de evasão mostram uma correlação negativa com valor de -0,166. Como em todas as turmas a relação $\mathrm{P} / \mathrm{N}$ foi superior à 2,9013 (o valor definido como limiar por Losada), estes resultados nos permitem concluir que, uma vez superada a taxa crítica de positividade e negatividade, valores mais elevados na relação $\mathrm{P} / \mathrm{N}$ não tem grande efeito correspondente na taxa de evasão.

Uma vez que a quantidade e a qualidade de postagens pelos alunos não mostrou ser um bom indicador para predizer a probabilidade de evasão foram então analisadas as postagens dos tutores. A avaliação do estilo de participação do tutor utilizou a escala apresentada na tabela 7. Nesta tabela considerou-se as categorias de 2 até 6 qualificavam-se como manifestações instigadoras de reflexão e de reação.

\begin{tabular}{|l|l|}
\hline $\begin{array}{c}\text { Categoria de } \\
\text { intervenção }\end{array}$ & \multicolumn{1}{c|}{ Descrição do tipo de intervenção } \\
\hline o & Respostas diretas a perguntas dos alunos. \\
\hline $\mathbf{1}$ & Manifestações positivas de aprovação a alguma contribuição de aluno. \\
\hline $\mathbf{2}$ & Perguntas de esclarecimento. \\
\hline 3 & Perguntas que verificam suposições. \\
\hline 4 & Perguntas que verificam evidências e linhas de raciocínio. \\
\hline 5 & Perguntas sobre pontos de vista ou perspectivas. \\
\hline 6 & Perguntas que verificam implicações e consequências. \\
\hline
\end{tabular}

Tabela 7. Estilo de intervenção do tutor 
Um exemplo de manifestações na categoria instigadora de reflexão seria uma postagem com o seguinte conteúdo:

É isso aí! Precisamos refletir e muito sobre o que assistimos, ou melhor, o que nossos alunos assistem. Todos concordam? O que pensam a respeito? Aguardamos os comentários.

Os resultados obtidos, pela análise dos mesmos dois fóruns foram os apresentados no gráfico 3 e detalhados na tabela 8 .

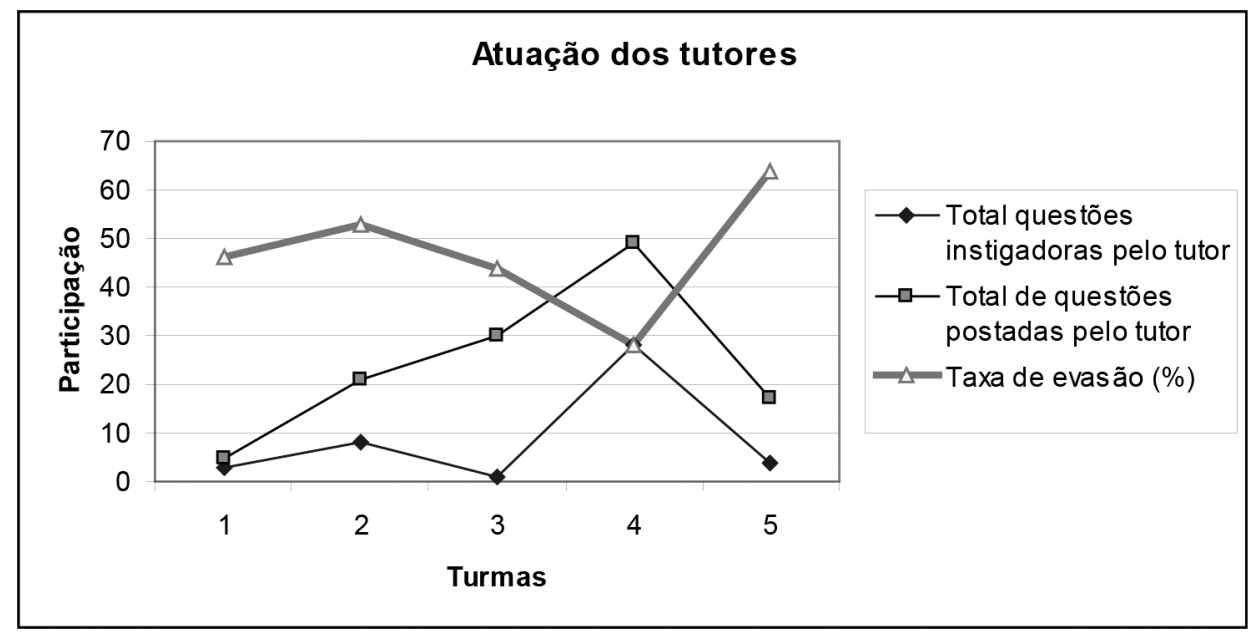

Gráfico 3. Tipos de intervenção dos tutores e evasão

\begin{tabular}{|l|l|l|l|l|l|l|}
\hline \multicolumn{1}{|c|}{ Atuação dos tutores } & \multicolumn{1}{|c|}{ T1 } & \multicolumn{1}{|c|}{ T2 } & \multicolumn{1}{|c|}{ T3 } & \multicolumn{1}{|c|}{ T4 } & \multicolumn{1}{|c|}{ T5 } & TOTAL \\
\hline Total questões instigadoras pelo tutor & 3 & 8 & $\mathbf{1}$ & $\mathbf{2 8}$ & 4 & 44 \\
\hline Total de questões postadas pelo tutor & 5 & 21 & 30 & 49 & 17 & 122 \\
\hline Taxa de evasão (\%) & 46 & 53 & 44 & 28 & 64 & 47 \\
\hline Média da turma (o-10) & 4,87 & 5,45 & 5,20 & 6,81 & 1,98 & 4,86 \\
\hline
\end{tabular}

Tabela 8. Estilo de participação do tutor e seu impacto na evasão e no desempenho

Estabelecendo a correlação entre estes indicadores relativos à atuação dos tutores, os indicadores derivados do desempenho e a evasão pelos alunos observa-se grande interrelação entre eles. A tabela 9 mostra os valores de correlação obtidos. 


\begin{tabular}{|l|c|}
\hline $\begin{array}{l}\text { Correlação entre a média da turma e a quantidade de postagens instigadoras } \\
\text { postadas pelo tutor }\end{array}$ & 0,61 \\
\hline Correlação entre quantidade de postagens instigadoras e percentual de evasão & $-0,71$ \\
\hline $\begin{array}{l}\text { Correlação entre a média da turma e a quantidade de postagens postadas pelo } \\
\text { tutor }\end{array}$ & 0,59 \\
\hline Correlação entre quantidade de postagens e percentual de evasão & $-0,69$ \\
\hline
\end{tabular}

Tabela 9. Correlação do desempenho dos tutores e a evasão

Os valores para a correlação entre total de postagens instigadoras foram maiores do que no caso de postagens em geral o que salienta a importância de que o tutor procure permear sua participação no fórum com questões instigadoras que incitem os alunos à reflexão e à participação ativa, conforme já foi também enfatizado por So y Brusch (2008) e por Mazzolini y Madison (2007). O estilo de participação do tutor tem grande influência não apenas no resultado em termos de aprendizagem como também contribui para reduzir a evasão. Quanto mais o tutor se fizer presente no fórum maior será a qualidade da aprendizagem e menor a taxa de evasão, percebendo-se neste estudo que a participação com questões instigadoras teve mais impacto na redução da evasão do que de outros tipos de participação (tipos o e 1 na tabela 7).

\section{CONCLUSÕES}

A evasão é um dos problemas que atinge as Instituições de ensino. A busca da causa deste problema tem sido investigado em estudos e pesquisas educacionais (Fávero y Franco, 2006; Lassibille y Navarro, 2007; Lobo, Montejunas, Hipólipo y Lobo, 2007; Kingston, 2008). O abandono em cursos de Educação a Distância é um desafio a ser enfrentado pelas instituições provedoras deste tipo de curso. Os grandes desafios a ser enfrentados envolvem as questões sócio afetivas e a metodologia utilizada. Inerente às questões sócio-afetivas são as estratégias de contato e interação com os alunos conforme salientaram (Jones y Issroff, 2005; Girasoli y Hannafin, 2008; Jones y Issrof, 2008; Donnely y Roisin, 2010; Fiuza, 2012).

Os alunos que fazem cursos a distância, na sua grande maioria, têm uma característica em comum, a solidão. Se não houver uma boa interação entre os integrantes do curso com o aluno, este poderá sentir-se desatendido e abandonar o curso e é possível constatar que cursos totalmente a distância têm apresentado índices mais elevados de evasão. A realização de encontros presenciais constitui um estimulo para o desenvolvimento do sentimento de pertencimento do aluno 
àquela comunidade de aprendizagem e isto aumenta a motivação para continuar participando efetivamente no processo de aprendizagem, até sua conclusão (Kember, 2008). Esta motivação pode ser aumentada através da mediação pelo tutor e o impacto na redução das taxas de evasão pode ser comprovado no estudo apresentado neste trabalho.

A EAD tem um importante papel social ao ampliar o acesso à educação e o uso de cursos mistos (blended learning) em que encontros presenciais são realizados, em adição às atividades desenvolvidas a distância, e ambos são apoiados pela tecnologia (Smith, Keer y Valcke, 2008; Donnely, 2010). A educação à distância, sem os encontros presenciais, pode ensejar menor interação entre o professor e o aluno e entre os próprios alunos aumentando o risco da evasão. Com o uso de ambientes virtuais mais interativos, é possível reduzir estes índices de desistência (Hsiu-Li y HsiPeng, 2007; Donnely, 2010). A interatividade pode e deve estar presente inclusive no material educacional o qual deve ser criado usando uma abordagem dialógica onde o texto busca instigar reflexão. Todavia, a possibilidade de interação entre as pessoas que participam do curso não pode ser totalmente substituída pelo texto por mais interativo e permeado de recursos multimídia que seja seja. Em decorrência, o papel da mediação do tutor no ambiente virtual de aprendizagem é vital e ele precisa procurar conhecer a realidade de seus alunos para poder interagir de forma mais contextualizada buscando instigar a reflexão sobre o tema em foco no curso. A ação educativa mais eficaz é precedida de reflexão e análise do meio de vida concreto do aluno (Sapiro, Feltovich, Jacobson y Coulson, 1995; May, So, Wong y Sculli, 2005; Masiello, Ramberg y Lanka, 2005).

Para Harasim (2006), da Simon Fraser University, de Vancouver, a alta taxa de desistência na Educação a Distância (80\% a 90\%) refere-se "às formas nãocolaborativas". Para a autora, a Educação a Distância é separada em três tipos: (1) colaborativo, o curso é on-line, com material impresso e o professor realiza atividades via internet com os alunos que debatem em chats e por e-mail; (2) a distância, com material impresso e professor; (3) treinamento com base em computador, isto é, cursos prontos que utilizam multimídia e não têm professores ou tutores para guiar os alunos. Ela defende o método colaborativo, pois nesta modalidade a taxa de evasão é a menor.

Assim Jeong y Joung (2007) num estudo com 38 estudantes universitários, avaliaram o quanto a anotação sobre o estilo de comunicação influenciou a estratégia de argumentação numa discussão on-line assíncrona. $O$ fato de algumas mensagens terem sido rotuladas influenciou a forma como impactaram o grupo. Mensagens rotuladas como críticas não tiveram continuidade enquanto que mensagens 
rotuladas como argumentos foram seguidas por outras mensagens também rotuladas da mesma forma trazendo evidências complementares para o debate. A crítica, tendo uma conotação mais negativa, não provocou o engajamento dos estudantes no debate o que corrobora os achados de Fredrickson (1998). Conforme defendido por Fredrickson y Losada (2005), equipes de alta performance trabalham com uma relação entre manifestações positivas e negativas maior do 2,9013. No presente estudo, a relação $\mathrm{P} / \mathrm{N}$ foi bastante superior ao limiar estabelecido por Losada (1999), em todas as turmas mesmo naquelas em que foi observada maior taxa de evasão o que nos permite concluir que embora uma relação $\mathrm{P} / \mathrm{N}$ maior do que o limiar da linha de Losada $(2,9013)$ deva existir para não criar um ambiente hostil no grupo o que reduziria a motivação para a continuidade no curso, isto não basta. Uma participação do tutor que, embora responda às perguntas dos alunos e seja permeada de manifestações positivas em relação às postagens dos alunos, não instigue reflexões e não chame uma maior participação dos integrantes do grupo, pode levar a um aumento na taxa de evasão, conforme foi constatado nas turmas 2 e 5 do curso analisado.

Segundo Kingston (2008) as instituições públicas e privadas, de modo geral, referem como a principal razão de evasão a falta de recursos financeiros para o aluno continuar seus estudos. Este também é o motivo que o aluno normalmente declara quando é questionado sobre seu abandono. No entanto o que se verifica, é que outras questões de ordem acadêmica, tais como as expectativas do aluno em relação à sua formação e sua própria integração com a Instituição, impactam sua decisão de abandonar um curso. Existindo a motivação o aluno pode passar a priorizar o investimento de tempo ou financeiro para a conclusão do curso.

Neste estudo, o índice de evasão no curso analisado foi de $47 \%$. Este índice é semelhante ao da média brasileira e de outros países sejam eles desenvolvidos ou não. Os motivos alegados para a evasão foram primeiramente a falta de tempo, seguido da organização pessoal em conciliar o curso e outras atividades. Os problemas de saúde e freqüência em outro curso também foram fatores relevantes neste estudo. Outros fatores também apontados foram a adaptação à EAD, a forma de apresentação do conteúdo do curso, dificuldades de interação com o professor, o excesso de conteúdo, a falta de interesse em estudar nesta modalidade, problemas administrativos e tecnológicos relacionados à disponibilidade do acesso ao material e tarefas do curso. Todavia a grande variação na taxa de evasão que ficou em $28 \%$ no melhor caso e em $64 \%$ no pior caso quando correlacionada com o estilo de mediação do tutor destacou a importância de sua participação permeada de questões instigantes, que incitem à reflexão e à participação continuada dos alunos ao longo do curso. 
Este estudo confirmou que o problema da evasão é uma realidade em nosso meio. Os contatos feitos com os alunos que evadiram permitiram elicitar as causas mais comuns para a evasão, os indicadores investigados mostraram-se capazes de sugerir estratégias para detectar os que estão prestes a evadir e tentar diminuir a evasão nos cursos do ensino a distância mediante uma participação do tutor mais instigadora.

Outras estratégias para diminuir a evasão passam pela conscientização do aluno em relação às demandas de um curso a distância tais como necessidade de maior autonomia do aluno, de auto-organização em relação ao uso do tempo e disponibilidade para participar ativamente das atividade colaborativas do curso. Por outro lado, mais importante ainda é capacitar os professores de forma a privilegiar ações do estar junto virtual, estimular a interação entre todos os participantes, pois o diálogo aproxima, criando um ambiente motivador e instigador para aumentar o tempo de dedicação e interação no curso pelos alunos.

Como estratégias para detectar o aluno prestes a evadir sugere-se um processo de acompanhamento contínuo e permanente do aluno. É preciso um monitoramento constante de sua satisfação, bem estar, adaptação, interação entre outros. Este acompanhamento busca criar uma base para planejar e desencadear ações que de um lado, assegurem que o aluno ajuste suas expectativas e o esforço que precisa dedicar às condições do curso que pretende realizar, mas de outro lado que os professores, tutores e demais integrantes da equipe docente atuando no curso busquem aprimorar a mediação.

\section{REFERÊNCIAS BIBLIOGRÁFICAS}

ABED (2013). Relatório Analítico da Educação a Distância no Brasil. Brasil: Pearson editora.

ABRAEAD (2007). Anuário Brasileiro Estatístico de Educação Aberta e a Distância. São Paulo: Instituto Monitor.

ABRAEAD (2008). Anuário Brasileiro Estatístico de Educação Aberta e a Distância. São Paulo: Instituto Monitor.

Araujo, L.; Lucena, G. (2005). Comunidades virtuais de aprendizagem: novas dinâmicas de aprendizagem exigem novas formas de avaliação. XVI simpósio Brasileiro de Informática na Educação. SBC/ UFJF, Juiz de Fora, MG.
Baxter, J. (2012). Who am I and what keeps me going? profiling the distance learning student in higher education. International Review of Research in Open and Distance Learning, 13 (4), (107-129).

Beckett, G. H.; Amaro-Jiménezb, C.; Beckettc, K. S. (2010). Students' use of asynchronous discussions for academic discourse socialization. Distance Education, 31 (3), (315-335).

Cetic (2012). Pesquisa sobre o uso das tecnologias de informação e comunicação no Brasil: TIC Educação 2011. Barbosa, A. Comitê Gestor da Internet no Brasil. 
Cheng, J.; Kulkarni, C.; Klemmer, S. (2013). Tools for predicting drop-off in large online classes. Paper presented at the Proceedings of the ACM Conference on Computer Supported Cooperative Work, CSCW, (121-124).

Cordero, J. M. (2005). Oficina Metodológica de Educação a Distância. [en línea] Disponível em: http://www.unitins.br [consulta 2012, 22 de diciembre].

Costa, A. C. C. (2013). Interacciones entre alumnos en entornos mediados por TIC. Un análisis de la dimensión social de los intercambios. RIED. Revista Iberoamericana de Educación a Distancia, 16 (1), (85-107).

De Wever, B.; Schellens, T.; Valck, M.; Van Keer, H. (2006). Content analysis schemes to analyze transcripts of on line asynchronous discursion groups: a review. Computers \& Education, 46 (1), (6-28).

Dingus, L. P.; Ellis, T. (2005). Using data mining as a strategy for assissing asynchronous discussion foruns, Computers \& Education, 45 (1), (141-160).

Donald, J. G. (2002). Learning to think: Disciplinary Perspectives. San Francisco: Jossey-Bass.

Donnely, R. (2010). Harmonizing technology with interaction in blended problembased learning. Computers \& Education, $54,(350-359)$.

Dowden, T.; Pittaway, S.; Yost, H.; McCarthy, R. (2013). Students' perceptions of written feedback in teacher education: Ideally feedback is a continuing twoway communication that encourages progress. Assessment and Evaluation in Higher Education, 38 (3), (349-362).

Fávero, R. V. M.; Franco, S. R. K. (2006). Um estudo sobre a permanência e a evasão na Educação a Distância. Revista Novas Tecnologias na Educação (CINTEDUFRGS), 4 (2).

Fidas, C. C.; Komis, V.; Tzawavaris, S.; Avouris, N. (2005). Heterogeneity of learning material in synchronous computer-supported collaborative modeling, Computers \& Education, 44 (2), (135-154).

Fredrickson, B. L. (1998). What good are positive emotions? Review of General Psychology, 2, (300-319).

Fiuza, P. J. (2012). Adesão e permanência discente na educação a distância: investigação de motivos e análise de preditores sociodemográficos, motivacionais e de personalidade para o desempenho na modalidade. Universidade Federal do Rio Grande do Sul. Instituto de Psicologia. Curso de Pós-Graduação em Psicologia. [en línea] Disponível em: http:// hdl.handle.net/10183/55089 [consulta 2012, 22 de diciembre].

Flach, L.; Antonello, C. S. (2011). Organizações culturais e a aprendizagem baseada em práticas. Cadernos EBAPE.BR. Rio de Janeiro, 9 (1), (155-175).

Fredrickson, B. L.; Losada, M. F. (2005). Positive affect and the complex dynamics of human flourishing. American Psychologist, 6o (7), (678-686).

Freire. P. (2003). À sombra desta mangueira. São Paulo: Olho d'Água.

Freire, P. (2006). Pedagogy of the oppressed. The Continuum International Group Inc, New York.

Girasoli, A. J.; Hannafin, R. D. (2008). Using asynchronous AV Communications Tools to increase academic sel-efficacy. Computers \& Education, 51, (16761682).

Grau-Valldosera, J.; Minguillón, J. (2011). Redefining dropping out in online higher education: A case study from the UOC. Paper presented at the ACM International Conference Proceeding Series, (7580).

Harasim, L. (2006). A History of E-learning: Shift happened. The International Handbook of virtual learning environments. Springer Netherlands, (59-94).

Heller, R. S.; Martin, C. D.; Haneef, N.; Gievska-Krliu, S. (2001). Using a theoretical 
multimedia taxonomy framework. Journal of Educational Resources in Computing, 1 (1), (6).

Heller, R. S. (2001). Use of the multimedia taxonomy for a research direction into design and evaluation of material for the elderly. Proceedings of the $2001 \mathrm{EC} / \mathrm{NSF}$ Workshop on Universal Accessibility of Ubiquitous Computing, (28-30).

Hsiu-Li, L.; Hsi-Peng, L. (2008). The role of experience and innovation characteristics in the adoption and continued use of e-learning websites. Computers \& Education, 51, (1405-1416).

Hung, S.; Cheng, M. (2013). Are you ready for knowledge sharing? An empirical study of virtual communities. Computers \& Education, 62, (8-17).

Jeon, A.; Joung, S. (2007). Scaffolding collaborative argumentation in asynchronous discussions with message constraints and message labels, Computers \& Education, 48 (3), (427-445).

Jones, A.; Issroff, K. (2005). Learning technologyes: Affective and social issues in computer-suported collaborative learning. Computers \& Education, 44 (4), (395-408).

Kember, D. (2008). The importance of establishing relevance in motivating student learning. Active Learning in High Education, 9 (3), (249-263).

Kingston, E. (2008). Emotional competence and drop-out rates in higher education. Esmerald Group Publishing Limited, 50 (2), (128-139).

Kolb, D. A.; Fry, R. (1975). Toward an Applied Theory of Experiential Learning. In: Cooper, C. L. Theories of Group Processes. London-Toronto: John Wiley and Sons.

Kolb, D. A. (1984). Experiential learning: experience as the source of learning and development. Englewood Cliffs: Prentice-Hall.

Lassibille, G.; Navarro, L. G. (2007). Why do higher education students drop out?
Evidence from Spain. Education Economics, 16 (1), (89-105).

Leeds, E.; Campbell, S.; Baker, H.; Ali, R.; Brawley, D.; Crisp, J. (2013). The impact of student retention strategies: An empirical study. International Journal of Management in Education, 7 (1-2), (22-43).

Leonhardt, M.; Tarouco, L.; Vicari, R.; Santos, E.; Silva, M. (2007). Using Chatbots for Network Management Training through Problem-based Oriented Education. Seventh IEEE International Conference on Advanced Learning Technologies (ICALT 2007), 5. Niigata, Japão. International, (845-847).

Lima, M.; Tarouco, L. (2004). Individual behavior in digital/virtual environments. The $8^{\text {th }}$ International Conference on Computer Supported Cooperative Work in Design, Xiamen - China. International Academic Publishers, v.2, (605-610).

Lima, M.; Tarouco, L. (2005). The use of groups in virtual/digital environment. Fifth IEEE International Conference on Advanced Learning Technologies, Kaohsiung - Taiwan, 1, (834-836).

Lobo, R.; Motejunas, P. R.; Hipólito, O.; Lobo, M. B. C. M. (2007). Dropping out in brazilian undergraduate courses. $\mathrm{Ca}$ dernos de Pesquisa. Fundação Carlos Chagas, São Paulo, 37 (132), (641-659).

Losada, M. (1999). The complex dynamics of high performance teams. Mathematical and Computer Modelling, 30 (9-10), (179-192).

Lykourentzou, I.; Giannoukos, I.; Nikolopoulos, V.; Mpardis, G.; Loumos, V. (2009). Dropout prediction in e-learning courses through the combination of machine learning techniques. Computers and Education, 53 (3), (950-965).

McCarthy, B. (1986). The 4MAT system: teaching to learning styles with right/left mode techniques. Barrington: Excel.

Martin-Blas, T.; Serrano_Fernandez, A. (2009). The role of new Technologies in 
the learning process: moodle as a teaching tool in physics. Computers \& Education, 52 (1), (35-44).

Martín, M. A.; López, E. (2012). La sociedad de la información y la formación del profesorado. E-actividades y aprendizaje colaborativo. RIED. Revista Iberoamericana de Educación a Distancia, 15 (1), (15-35).

Masiello, I.; Ramberg, R.; Lonka, K. (2005). Attitudes to the application of a webbased learning system in a microbiology course. Computers \& Education, 45 (2), (171-185).

Mazzolini, M.; Madison, S. (2007). When to Jump in: the role of the instructor in online discussion forums. Computers \& Education, 49, (193-213).

May So, W. C.; Wong, D.; Sculli, D. (2005). Factors affecting intentions to purchase via the Iinternet. Industrial Management \& Data Systems, 105 (9), (12251234).

Moore, M.; Kearsley, G. (2007). Educação a Distância: uma visão integrada. São Paulo: Thomson Learning.

Morin, A. E. (2003). Introdução ao pensamento complexo. Lisboa: Instituto Piaget.

Nistor, N.; Neubauer, K. (2010). From participation to dropout: Quantitative participation patterns in online university courses. Computers and Education, 55, (2), (663-672).

Offir, B.; Lev, Y.; Bezale, R. (2008). Surface and deep learning processes in distance education: Synchronous versus Asynchronous Systems. Computers \& Education, 51, (1172-1183).

Park, J. H.; Choi, H. J. (2009). Factors influencing adult learners' decision to drop out or persist in online learning. Educational Technology and Society, 12 (4), (207-217).

Regan, K.; Evmenova, A.; Baker, P.; Jerome, M. K.; Spencer, V.; Lawson, H.; Werner, T. (2012) Experiences of instructors in online learning environments: Identifying and regulating emotions. Internet and Higher Education, 15, (3), (204212).

Santos, E. M.; Tomotake, M. E.; Oliveira Neto, J. D.; Cazarini, E. W.; Araújo, E. M.; Oliveira, S. R. M. (2008). Evasão na Educação a Distância: identificando causas e propondo estratégias de prevenção. São Paulo.

Sapiro, R.; Feltovich, P.; Jacobson, M.; Coulson, R. (1995). Using the Brain in CaseBased Courses.

Smith, M.; Keer, H.; Valcke, M. (2008). Blending asynchronous discussion groups and peer tutoring in higher education: An exploratory study of online peer tutoring behaviour. Computers \& Education, 5O, (207-223).

So, H. J.; Brush, T. A. (2008). Student perceptions of collaborative learning, social presence and satisfaction in a blended learning environment: Relationships and critical factors. Computers \& Education, 51, (318-336).

Tarouco, L.; Hack, L.; Vit, A.; Geller, M. (2000). Supporting group learning and assessment through Internet. TERENA Networking Conference, Lisboa Proceedings TERENA Networking Conference 2000. Amsterdan: TERENA Secretariat.

Tarouco, L.; Vicari, R.; Geller, M.; Meirelles. L. (2003). Cooperative learning in a virtual reality environment. IADIS International e-Society Conference, 1. Lisboa, Portugal: International Association for the development of the information society, (171-178).

Tarouco, L. (2004). Interactively Building a Knowledge Base for a Virtual Tutor. Lecture Notes in Computer Science, 3220. Heidelberg, (904-905).

Tinto, V. (1975). Dropout from higher education: a theoretical synthesis of recent research. Review of Educational Research, 45 (1), (89-125). 
Tinto, V. (1987). Leaving college. Chicago: University of Chicago Press.

Tinto, V. (1993). Leaving college: rethinking the causes and cures of student attrition. Chicago: University of Chicago Press.

Underwood, J. D. M. (1998). Integrated learning systems: where does the management take place? Education and Information Technologies, 2 (4), (275-286).

Underwood, G.; Underwood, J. D. M. (1998). Children's interactions and learning outcomes with interactive talking books. Computers \& Education, 30, (1-2), (95102).

Vercellone-Smith, P.; Jablokow, K.; Friedel, C. (2012). Characterizing communication networks in a web-based classroom: Cognitive styles and linguistic behavior of self-organizing groups in online dis- cussions. Computers and Education, 59 (2), (222-235).

Vit, R.; Tarouco, L. M. R. (2000). Group Learning Trough Internet. SITE'20oo 11th International Conference, San Diego, California - USA. Charlottesville, VA - USA: AACE, (143 - 147).

Vygotsky, L. S. (2008). Mind in society: The development of higher psychological processes. Cambridge, MA: Harvard University Press.

Walraven, A.; Brand-Gruwel, S.; Boshuizen, H. P. A. (2009). How students evaluate information and sources when searching the Word Wide Web for information. Computers \& Education, Elsevier. 52, (234-246).

\section{PERFIL ACADÊMICO E PROFISSIONAL DOS AUTORES}

Adelina Mezzari. Graduada em Farmácia-Bioquímica (UFRGS). Atua como professora na Faculdade de Farmácia da UFRGS e na Universidade Federal de Ciências da Saúde de Porto Alegre (UFCSPA). Suas áreas de interesse são: Ensino e Pesquisa em Educação e Micologia.

E-mail: mezzari@ufcspa.edu.br

Liane Margarida Rockenbach Tarouco. Graduada em Física (UFRGS), MSc em Ciência da Computação (UFRGS), Dr Engenharia Elétrica (USP). Atua como professora na Faculdade de Educação e nos programas de pós-graduação em Ciência da Computação e Informática na Educação da UFRGS. Suas áreas de interesse são: Redes de Computadores, Tecnologia da Informação e Comunicação na Educação, Educação a distância.

E-mail: liane@penta.ufrgs.br 
Ana Marli Bulegon. Graduada em Matemática (UNIFRA), Mestre em Ensino de Física e de Matemática (UNIFRA), Doutora em Informática na Educação (UFRGS). Atua como professora no Mestrado Profissional em Ensino de Física e de Matemática e em cursos de Graduação do Centro Universitário Franciscano (UNIFRA). Áreas de interesse: Ensino de Física e de Matemática, Tecnologia da Informação e Comunicação na Educação, Educação a distância.

E-mail: anabulegon@gmail.com

Rute Vera Maria Favero. Graduada em Tecnólogo em Processamento de Dados (UNISINOS), MSc em Educação (UFRGS), Doutoranda em Educação (UFRGS). É coordenadora do Suporte Pedagógico às Plataformas de EAD da UFRGS e é professora no Colégio de Aplicação (UFRGS). Suas áreas de interesse são: Educação a Distância, Tecnologia da Informação e Comunicação na Educação, Redes Sociais, Educação e Construção de Conhecimento.

E-mail: rute@ufrgs.br

Barbara Gorziza Avila. Graduada em Matemática-licenciatura (UFRGS). Atua como estudante de doutorado no Programa de Pós-Graduação em Informática na Educação (UFRGS). Suas áreas de interesse são: Mundos virtuais no contexto de ensino-aprendizagem e Educação a Distância.

E-mail: barbaragorzizaavila@gmail.com

Geraldo Ribas Machado. Graduado em Administração de Empresas (PUCRS), Mestre em Engenharia da Produção (UFRGS) e Doutor em Educação (UFRGS). Atua como professor do Departamento de Ciências da Informação da Faculdade de Biblioteconomia e Comunicação da UFRGS. Suas áreas de interesse são Probabilidade e Estatística, Avaliação Institucional.Educação (UFRGS). Mestre em Engenharia da Produção (UFRGS). Atua como professor do Departamento de Ciências da Informação na Faculdade Biblioteconomia e Comunicação UFRGS. Pesquisador dos Grupos de Pesquisa CNPq: Grupo de Estudos Inovação e Avaliação na Universidade (UFRGS), TEIAS - Tecnologia em Educação para Inclusão e Aprendizagem em Sociedade (UFRGS).

E-mail: geraldo.machado@ufrgs.br 


\title{
DIRECCIÓN DE LOS AUTORES
}

\author{
Adelina Mezzari \\ Faculdade de Farmácia da Universidade Federal do \\ Rio Grande do Sul (UFRGS) \\ Av. Ipiranga, 2752 \\ CEP: 90610-00o, Porto Alegre \\ Rio Grande do Sul, Brasil
}

Fechas de recepción del artículo: 03/11/12

Fecha de aceptación del artículo: 14/03/13

\section{Como citar este artículo:}

Mezzari, A.; Rockenbach Tarouco, L. M.; Gorziza Avila, B.; Ribas Machado, G.; Maria Favero, R. V.; Marli Bulegon, A. (2013). Estratégias para detecção precoce de propensão à evasão. RIED. Revista Iberoamericana de Educación a Distancia, volumen $16, \mathrm{n}^{\circ} 2$, pp. 147-175. 